\title{
Efficacy and Safety of Nebivolol and Rosuvastatin Combination Treatment in Patients with Concomitant Hypertension and Hyperlipidemia
}

This article was published in the following Dove Press journal:

Drug Design, Development and Therapy

\author{
Moo-Yong Rhee (1D \\ Cheol Ho Kim ${ }^{2}$ \\ Youngkeun Ahn $\mathbb{D}^{3}$ \\ Joon-Han Shin (1D ${ }^{4}$ \\ Seung Hwan Han (1D) \\ Hyun-Jae Kang (iD ${ }^{6}$ \\ Soon Jun Hong $\mathbb{1}^{7}$ \\ Hae-Young $\operatorname{Kim} \mathbb{1}^{8}$ \\ 'Cardiovascular Center, Dongguk \\ University Ilsan Hospital, Goyang, \\ Republic of Korea; ${ }^{2}$ Department of \\ Internal Medicine, Seoul National \\ University Bundang Hospital, Seongnam, \\ Republic of Korea; ${ }^{3}$ Division of \\ Cardiology, Department of Internal \\ Medicine, Chonnam National University \\ Hospital, Gwangju, Republic of Korea; \\ ${ }^{4}$ Department of Cardiology, Ajou \\ University Medical Center, Suwon, \\ Republic of Korea; ${ }^{5}$ Division of \\ Cardiology, Department of Internal \\ Medicine, Gachon University Gil Medical \\ Center, Incheon, Republic of Korea; \\ ${ }^{6}$ Department of Internal Medicine, Seoul \\ National University Cardiovascular \\ Center, Seoul National University \\ Hospital, Seoul, Republic of Korea; \\ ${ }^{7}$ Department of Cardiology, \\ Cardiovascular Center, Korea University \\ Anam Hospital, Seoul, Republic of Korea; \\ ${ }^{8}$ Department of Health Policy and \\ Management, College of Health Science \& \\ Department of Public Health Science, \\ Graduate School, Korea University, \\ Seoul, Republic of Korea
}

Correspondence: Cheol Ho Kim Department of Internal Medicine, Seoul National University Bundang Hospital, Seongnam, Republic of Korea

Tel +82 3I 787700 I

Fax +82 3I 787405 I

Email cheolkim55@gmail.com
Purpose: We evaluated the efficacy and safety of nebivolol and rosuvastatin combination treatment in patients with hypertension and hyperlipidemia.

Patients and Methods: Eligible patients, after more than 4 weeks of therapeutic lifestyle change, were randomly assigned to three groups: $5 \mathrm{mg}$ nebivolol plus $20 \mathrm{mg}$ rosuvastatin (NEBI/RSV), $20 \mathrm{mg}$ rosuvastatin (RSV), or $5 \mathrm{mg}$ nebivolol (NEBI). Treatments lasted 8 weeks.

Results: Efficacy was analyzed using data from 276 patients. Sitting systolic and diastolic blood pressures differed between the NEBI/RSV and RSV groups (LSmean difference = -5.89 and $-5.99 \mathrm{mmHg}$; $95 \%$ confidence interval $[\mathrm{CI}]=-9.88$ to $-1.90 \mathrm{mmHg}$ and -8.13 to $-3.84 \mathrm{mmHg}$, respectively). Reductions in the two pressures did not differ between the NEB/ RSV and NEB groups. The percent reduction in low-density lipoprotein (LDL) cholesterol differed between the NEBI/RSV and NEBI groups (LSmean difference $=-47.76 \%, 95 \% \mathrm{CI}$ $=-52.69$ to $-42.84 \%$ ) but not between the NEBI/RSV and RSV groups. The blood pressure (BP) control rate was higher in the NEBI/RSV group than in the RVS group $(51.09 \%$ vs $29.67 \%, p=0.003)$. The LDL cholesterol goal achievement rate was higher in the NEBI/ RSV group than in the NEBI group $(85.87 \%$ vs $11.83 \%, p<0.001)$. The incidence of adverse drug reactions in the NEBI/RSV, RSV, and NEBI groups was $8.51 \%, 7.45 \%$, and $8.60 \%$, respectively $(p=0.950)$.

Conclusion: Nebivolol plus rosuvastatin treatment is effective in reducing BP and LDL cholesterol levels and is safe in patients with hypertension and hypercholesterolemia without the loss of BP or the LDL cholesterol-lowering effect of each drug.

Trial Registration: CRIS registration number KCT0002148.

Keywords: hypertension, hypercholesterolemia, nebivolol, rosuvastatin

\section{Introduction}

Various risk factors affecting cardiovascular disease often exist simultaneously. Among modifiable risk factors, hypertension and hypercholesterolemia commonly coexist. ${ }^{1}$ The coexistence of hypertension and hyperlipidemia has been shown to exert detrimental effects on cardiovascular outcomes, ${ }^{2}$ and treatment of both diseases simultaneously has been shown to be four times more effective in lowering cardiovascular events than treating only one disease. ${ }^{3}$

As a treatment of hypercholesterolemia, lowering low-density lipoprotein (LDL) cholesterol levels with statins is effective for both primary and secondary prevention of cardiovascular events. ${ }^{4}$ Among antihypertensive drugs, beta-blockers are not 
recommended as an initial drug for treating uncomplicated hypertension in some guidelines because of their inferior outcomes compared with other antihypertensive drug classes. ${ }^{5,6}$ The adverse effects of beta-blockers on lipid and glucose metabolism have been considered to offset their beneficial effect in lowering blood pressure (BP). ${ }^{7}$ However, beta-blockers are important and essential drugs in treating hypertensive patients with coronary heart disease, heart failure, and atrial fibrillation, and are recommended as a first-line drug in these patients. ${ }^{8}$ Therefore, beta-blockers and statins may be co-administered in patients with hypertensive cardiovascular disease and hypercholesterolemia.

Different from other beta-blockers, nebivolol, a thirdgeneration beta-blocker, has a better metabolic profile, ${ }^{7}$ along with a nitric oxide-mediated vasodilatory property. ${ }^{9}$ However, no study has evaluated whether combining nebivolol plus statin treatment has the same effect as statins or nebivolol alone treatment on lipid parameters and BP. In the present study, we evaluated the efficacy of combining nebivolol plus rosuvastatin treatment on BP and lipid parameters and its safety in patients with concomitant hypertension and hyperlipidemia.

\section{Patients and Methods}

\section{Study Design}

This phase-III, randomized, double-blind, parallel-group trial involving patients with hypertension and hypercholesterolemia was conducted at 27 centers across Korea from September 2016 through September 2018. The Institutional Review Board of each participating institution (supplementary Table S7) and the Ministry of Food and Drug Safety approved the study protocol. Written informed consent was provided by all study participants. This study was conducted in accordance with the Declaration of Helsinki.

After eligibility screening, participants entered therapeutic lifestyle change (TLC) for $>4$ weeks. The duration of the TLC was $\geq 6$ weeks for participants receiving fibrates. Detailed education on TLC was provided by study coordinators. During the TLC, all lipid-modifying and antihypertensive medications were discontinued. After 4- or 6-weeks TLC, participants that meet the randomization inclusion criteria were randomly assigned at a 1:1:1 ratio to one of the following groups: the NEBI/RSV group (5 mg nebivolol and $20 \mathrm{mg}$ rosuvastatin daily for 8 weeks), the RSV group (20 $\mathrm{mg}$ rosuvastatin daily for 8 weeks), and the NEBI group
(5 mg nebivolol daily for 8 weeks). Elyson Pharmaceutical Co., Ltd. supplied nebivolol (Menarini Korea, Seoul, Korea) and rosuvastatin (AstraZeneca Korea, Seoul, Korea). For double blinding, each study drug and placebo drug of identical appearance were packed in blisters and supplied to patients.

All participants were instructed to take the assigned study drugs once daily every morning for the study duration. Prior to each scheduled visit, patients were instructed to fast for at least $8 \mathrm{~h}$ and not to take the study drugs in the morning. At each visit, after 5 min of rest, the sitting BP was measured three times at 2-min intervals using a validated oscillometric device (WatchBP Home, Microlife AG, Widnau, Switzerland). Three readings of the sitting systolic BP (sitSBP) and the diastolic BP (sitDBP) were averaged. The arm with the higher average sitSBP was determined as the index arm; the BP was obtained from the index arm during subsequent visits. At each visit, fasting blood samples were collected and sent to a central laboratory (GC LabCell, Yongin, Korea) to analyze total cholesterol, LDL cholesterol, high-density lipoprotein (HDL) cholesterol, triglyceride, apolipoprotein (Apo) A1, and ApoB levels. LDL-cholesterol levels were measured directly (LDL-C plus 2nd generation or LDLcholesterol Gen. 3, Roche, Mannheim, Germany).

\section{Study Population}

Inclusion criteria for randomization were as follows: participants (age, 20-79 years) with hypertension (systolic BP 140-179 mmHg and diastolic BP $\leq 109 \mathrm{mmHg}$, or currently receiving antihypertensive medications) and hypercholesterolemia (as defined according to the National Cholesterol Education Program Adult Panel III [NCEP-ATP III]).${ }^{10}$ Exclusion criteria included the following: LDL cholesterol levels $>250 \mathrm{mg} / \mathrm{dL}$ and/or triglyceride levels $\geq 400 \mathrm{mg} / \mathrm{dL}$ at randomization; a difference in repeatedly measured $\mathrm{BP}$ of the selected index arm at screening sitSBP $\geq 20 \mathrm{mmHg}$ or sitDBP $\geq 10 \mathrm{mmHg}$; symptomatic orthostatic hypotension; secondary hypertension; severe heart disease (NYHA class III-IV heart failure), clinically significant valvular disease of the heart, myocardial infarction and unstable angina; bradycardia $(<60$ bpm), second or third degree atrioventricular block; uncontrolled autoimmune diseases; bronchospasm or asthma; poorly controlled diabetes (HemoglobinA1c $\geq 9.0 \%$ ); uncontrolled thyroid disease (thyroid-stimulating hormone levels $\geq 1.5$ times of the normal upper limit); clinically significant renal (serum creatinine $\geq 2 \mathrm{mg} / \mathrm{dL}$ ) 
or hepatic diseases (aspartate transaminase or alanine transaminase $\geq 2$ times of the normal upper limit); surgical or medical disease that significantly affects absorption, distribution, metabolism, and elimination of study drugs; chronic inflammatory disease requiring chronic inflammatory treatment; history of myopathy, rhabdomyolysis, and/ or creatine phosphokinase $\geq 3$ times of the upper limit of normal; history of malignant tumors including leukemia and lymphoma in the past 5 years; clinical history of alcohol or drug abuse; hypersensitivity to investigational drugs; and women who were pregnant or breastfeeding, or could potentially become pregnant because of not using contraception throughout the study.

\section{Efficacy and Safety Assessments}

As a primary efficacy evaluation, (1) the change in sitSBP after 8-weeks treatment from baseline was compared between the NEBI/RSV and the RSV groups, and (2) the percent change in LDL cholesterol after 8-weeks treatment from baseline was compared between the NEBI/RSV and the NEBI groups.

Secondary efficacy was compared changes in sitDBP and the percent changes in total cholesterol, triglyceride, HDL cholesterol, response and control rates of BP, and achievement rate of LDL cholesterol goal after 8 weeks of treatment. The BP control rate was defined as the percentage of patients who reached a mean sitSBP $<140 \mathrm{mmHg}$ and sitDBP $<90 \mathrm{mmHg}$ after 8-weeks treatment. BP response rate was defined as the percentage of patients who reached a reduction in sitSBP $\geq 20 \mathrm{mmHg}$ or sitDBP $\geq 10 \mathrm{mmHg}$ from baseline values after 8 weeks of treatment. The LDL cholesterol goal achievement rate was calculated according to NCEP-ATP III guidelines (high risk: LDL-C level $<100 \mathrm{mg} / \mathrm{dL}$; moderate/moderately high risk: LDL-C level $<130 \mathrm{mg} / \mathrm{dL}$; low risk: LDL-C level $<160 \mathrm{mg} / \mathrm{dL}){ }^{10}$

Safety and tolerability were assessed by monitoring adverse events (AEs), serious AEs, and possible association of AEs with the study drugs and using laboratory tests.

\section{Sample Size}

Sample sizes were determined according to the differences in the changes in sitSBP and LDL cholesterol levels from baseline to week 8 between treatment and placebo groups.

Referring to previous reports, differences in the mean changes in sitSBP among the nebivolol and placebo groups were assumed to be -12.64 and $-5.28 \mathrm{mmHg}$, respectively; the common standard deviation was assumed to be $13.75 \mathrm{mmHg}^{11,12}$ To assess the difference in the changes of sitSBP, an adequate sample size was calculated as 74 subjects per group, with a one-sided significance level of $2.5 \%$ and a power level of $90 \%$.

The differences in mean percent change in LDL cholesterol in the rosuvastatin and placebo groups were assumed to be $-55 \%$ and $-7 \%$, respectively, with an assumed standard deviation of $11.1 \% .^{13,14}$ To assess the difference in the mean percent change of LDL cholesterol level, an adequate sample size was calculated to be three subjects per group.

From the two estimates, we selected a larger sample size of 74 subjects per group to achieve a sufficient level of statistical power to detect the efficacy of the testing treatment. Finally, 276 subjects (92 subjects in each group) were determined as the total sample size assuming a $20 \%$ drop-out rate.

\section{Statistical Analysis}

For efficacy analysis, we used a full-analysis set (FAS) that included all subjects who were administered the study drugs at least once after randomization, with at least one efficacy evaluation data after baseline used. In a perprotocol set (PPS), we included patients who completed the trial according to the protocol without significant violations that might affect efficacy outcomes. If any values in the primary and secondary efficacy points were missing, we used the last observation carried forward imputation method. Differences in primary efficacy outcomes between the treatment groups were evaluated by analysis of covariance (ANCOVA), using baseline values (for BP and LDL cholesterol levels) as covariates. If the upper limit of the $95 \%$ confidence interval $(\mathrm{CI})$ of the corrected least-square mean (LSmean) for the difference between the test and control groups was $<0$, the test drug was considered to be superior to the control. Differences in secondary efficacy outcomes within each treatment group were compared using a paired $t$-test or the Wilcoxon's signed-rank test. Differences in secondary efficacy outcomes between the treatment groups were evaluated using ANCOVA with baseline values as covariates.

The safety-analysis set (SAF) included patients who had received the study drugs at least once after randomization and had at least one safety assessment during the treatment period. All AEs were coded using the Medical Dictionary for Regulatory Activities (MedDRA; v 21.1). AE incidences were compared between groups using Pearson's chi-square or Fisher's exact tests. 
All statistical analyses were performed using $\operatorname{SAS}^{\circledR}$ software (v. 9.4; SAS Institute, Cary, NC, USA).

\section{Results}

\section{Patients' Disposition}

Of the 659 participants screened, 282 were randomly assigned to one of the three treatment groups. Among these, 281 were included in the SAF after excluding one participant who did not take the study drugs, and 276 were included in the FAS after further excluding five participants owing to missing the collection of the primary efficacy data. The mean patient age was $62.25 \pm 9.56$ years; most patients were men (75.7\%). Among the 276 participants, 29 were excluded from PPS for the following reasons: consent withdrawal $(\mathrm{n}=8)$, visit window violated $(\mathrm{n}$ $=9)$, AEs $(n=3)$, non-compliance to study drugs $(n=1)$, use of contraindicated drugs $(n=2)$, protocol violations ( $n$ $=3$ ), and other reasons $(\mathrm{n}=3)$ (Figure 1). The baseline characteristics of FAS are presented in Table 1.

\section{Efficacy Regarding BP Reduction}

The difference in BP reduction from baseline after 8 weeks of treatment was larger in the NEBI/RSV group than in the RVS group, but did not differ from that in the NEBI group (Table 2). The difference in sitSBP reduction from baseline after 8 weeks of treatment was significantly larger in the NEBI/RSV group than in the RSV group (LSmean difference $=-5.89$ $\mathrm{mmHg}$; $95 \% \mathrm{CI}=-9.88$ to $-1.90 \mathrm{mmHg}$ ), but did not differ from that in the NEBI group (LSmean difference $=1.85 ; 95 \%$ $\mathrm{CI}=-2.23$ to 5.93 ). Likewise, the difference in sitDBP reduction was significantly larger in the NEBI/RSV group than in the RSV treatment group (LSmean difference $=-5.99$ $\mathrm{mmHg}$; $95 \% \mathrm{CI}=-8.13$ to $-3.84 \mathrm{mmHg}$ ) but did not differ from that in the NEBI group (LSmean difference $=0.45$ $\mathrm{mmHg}$; $95 \% \mathrm{CI}=-1.72$ to $2.62 \mathrm{mmHg}$ ).

The BP control rate was $51.09 \%$ in the NEBI/RSV group, $29.67 \%$ in the RSV group, and $48.39 \%$ in the NEBI group (NEBI/RSV vs RSV, $p=0.003$; NEBI/RSV vs NEBI, $p=0.714$; Figure $2 \mathrm{~A}$ ). The BP response rate was $45.65 \%$ in the NEBI/RSV group, $24.18 \%$ in the RSV group, and $54.84 \%$ in the NEBI group (NEBI/RSV vs RSV, $p=0.002 ; \mathrm{NEBI} / \mathrm{RSV}$ vs NEBI, $p=0.212$; Figure 2B).

\section{Efficacy Regarding LDL Cholesterol Reduction}

The percent reduction in LDL cholesterol levels from baseline after 8 weeks of treatment in the NEBI/RSV group was greater compared to the NEBI group (LSmean difference $=-47.76 \% ; 95 \% \mathrm{CI}=-52.69$ to $-42.84 \%$ ), but

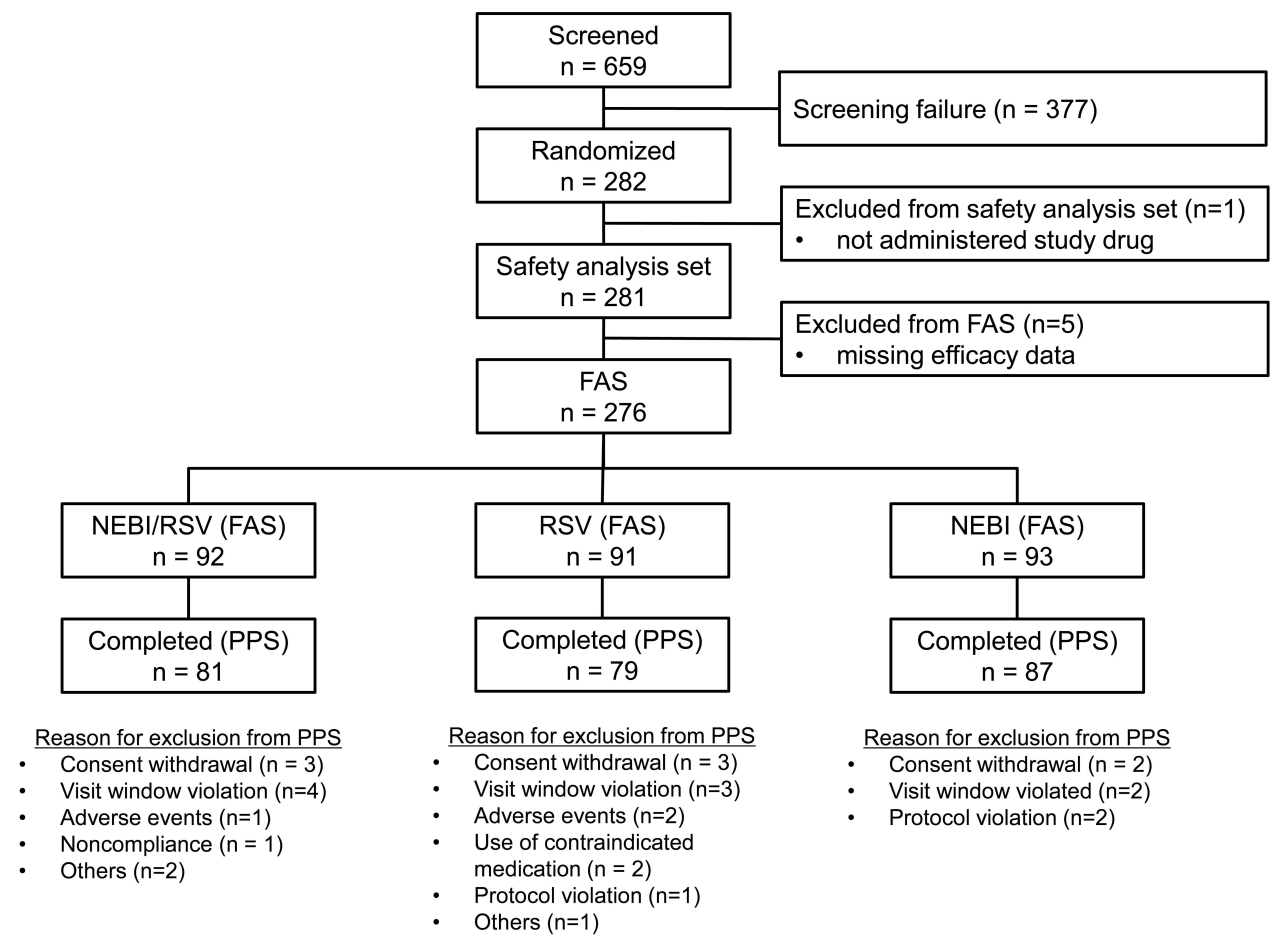

Figure I Participants disposition. 
Table I Baseline Characteristics of Study Population (FAS, n=276)

\begin{tabular}{|c|c|c|c|c|}
\hline & NEB/RSV & RSV & NEB & $\mathbf{p}$ \\
\hline $\mathrm{n}$ & 92 & 91 & 93 & \\
\hline Age, years (SD) & $62.76(9.52)$ & $61.74(9.75)$ & $62.24(9.49)$ & $0.915^{\mathrm{a}}$ \\
\hline Sex, men, n (\%) & $68(73.91)$ & $65(7 \mid .43)$ & $76(81.72)$ & $0.235^{\mathrm{b}}$ \\
\hline Body mass index, $\mathrm{kg} / \mathrm{m}^{2}(\mathrm{SD})$ & $43.59(13.42)$ & $42.78(6.25)$ & $42.98(5.64)$ & $0.416^{\mathrm{a}}$ \\
\hline \multicolumn{5}{|l|}{ Smoking, n (\%) } \\
\hline Never smoked & $33(35.87)$ & $28(30.77)$ & $29(31.18)$ & $0.723^{b}$ \\
\hline Ex-smoker & $38(4 \mid .30)$ & $35(38.46)$ & $35(37.63)$ & \\
\hline Current smoking & $21(22.83)$ & $28(30.77)$ & $29(31.18)$ & \\
\hline Drinking, n (\%) & $55(59.78)$ & $55(60.44)$ & $46(49.46)$ & $0.240^{\mathrm{b}}$ \\
\hline Diabetes, n (\%) & $22(23.91)$ & $15(16.48)$ & $18(19.35)$ & $0.447^{\mathrm{b}}$ \\
\hline eGFR, $\mathrm{mL} / \mathrm{min}$ per $1.73 \mathrm{~m}^{2}(\mathrm{SD})$ & 85.91 (13.42) & $88.90(16.11)$ & $88.46(12.96)$ & $0.173^{\mathrm{a}}$ \\
\hline \multicolumn{5}{|l|}{ Cardiovascular disease, n (\%) } \\
\hline Ischemic heart disease & $19(20.65)$ & $25(27.47)$ & $26(27.96)$ & $0.444^{\mathrm{b}}$ \\
\hline Peripheral vascular disease & $I(1.10)$ & $3(3.30)$ & $3(3.23)$ & $0.911^{c}$ \\
\hline Cerebrovascular disease & $2(2.17)$ & $2(2.20)$ & $7(7.53)$ & $0.121^{\mathrm{c}}$ \\
\hline \multicolumn{5}{|c|}{ Previous cardiovascular medication, n (\%) } \\
\hline Lipid modifying agents & $55(59.78)$ & $68(74.73)$ & $64(68.82)$ & $0.093^{b}$ \\
\hline ACE inhibitors or ARBs & $55(59.78)$ & $42(46.15)$ & $52(55.91)$ & $0.163^{b}$ \\
\hline Calcium channel blockers & $24(26.09)$ & $32(35.16)$ & $23(24.73)$ & $0.236^{\mathrm{b}}$ \\
\hline Beta-blocker & II (1I.96) & $16(17.58)$ & $14(15.05)$ & $0.563^{b}$ \\
\hline Cardiac drugs & II (II.96) & $6(6.59)$ & $10(10.75)$ & $0.440^{\mathrm{b}}$ \\
\hline Diuretics & $4(4.35)$ & $4(4.40)$ & $2(2.15)$ & $0.669^{c}$ \\
\hline Peripheral vasodilators & I (I.09) & I (I.I0) & I (I.08) & $1.000^{c}$ \\
\hline
\end{tabular}

Notes: Groups were compared by ${ }^{\mathrm{a} K r u s k a l-W a l l i s ~ t e s t, ~}{ }^{\mathrm{b}}$ Pearson's chi-square test and ${ }^{\mathrm{c}}$ Fisher's exact test. Cerebrovascular disease $=$ carotid artery stenosis + cerebral infarction + cerebral arteriosclerosis + cerebral hemorrhage + cerebellar infarction. Peripheral vascular disease $=$ peripheral vascular disorder + peripheral arterial occlusive disease + subclavian artery stenosis. Ischemic heart disease $=$ angina pectoris + angina unstable + myocardial infarction + acute myocardial infarction + coronary artery disease + Prinzmetal angina + myocardial ischemia.

Abbreviations: FAS, full analysis set; NEB/RSV, nebivolol $5 \mathrm{mg} /$ rosuvastatin $20 \mathrm{mg}$ treatment; RSV, rosuvastatin $20 \mathrm{mg}$ alone treatment; NEB, nebivolol $5 \mathrm{mg}$ alone treatment; eGFR, estimated glomerular filtration rate; ACE, angiotensin-converting enzyme; ARBs, angiotensin receptor blockers.

did not differ from that in the RSV group (LSmean difference $=1.50 \% ; 95 \% \mathrm{CI}=-3.05$ to $6.06 \%)($ Table 2$)$.

Changes in total cholesterol, HDL cholesterol, triglyceride, ApoA1, ApoB, ApoB/A1 ratio, and non-HDL cholesterol level are presented in Table 3. Percent reductions in total cholesterol and triglyceride, $\mathrm{ApoB}, \mathrm{ApoB} / \mathrm{A} 1$ ratio, and non-HDL cholesterol levels were larger in the NEBI/ RSV group than in the NEBI group but did not differ from that in the RSV group. HDL cholesterol and ApoA1 levels were increased in the NEBI/RSV group, but reduced in the NEBI group from baseline after 8 weeks of treatment. However, increase in HDL cholesterol and ApoA1 levels in the NEBI/RSV group were lower than those in the RSV group (LSmean difference for HDL cholesterol elevation = $-6.88 ; 95 \% \mathrm{CI}=-12.08$ to -1.67 , LSmean difference for ApoA1 elevation $=-3.71,95 \% \mathrm{CI}=-7.40$ to -0.02 ).
The goal achievement rate of LDL cholesterol levels was $85.87 \%$ in the NEBI/RSV group, $92.31 \%$ in the RSV group, and $11.83 \%$ in the NEBI group (NEBI/RSV vs RSV, $p=0.163$; NEBI/RSV vs NEBI, $p<0.001$; Figure 2C).

Results of subgroup analysis (age $\geq 65$ and $<65$ years, men and women, age $\geq 65$ vs $<65$ years, men vs women) are shown in supplementary Tables $\mathrm{S} 1-\underline{\mathrm{S}} 6$.

\section{Safety}

In the SAF $(\mathrm{n}=281)$, the incidences of AEs considered to be related to the study drugs were $8.51 \%$ in the NEBI/RSV group, $7.45 \%$ in the RSV group, and $8.60 \%$ in the NEBI group, but not significantly different among the treatment groups (Table 4, $p=0.950$ ). Among the study drug-related AEs, symptomatic bradycardia was found in two cases in 


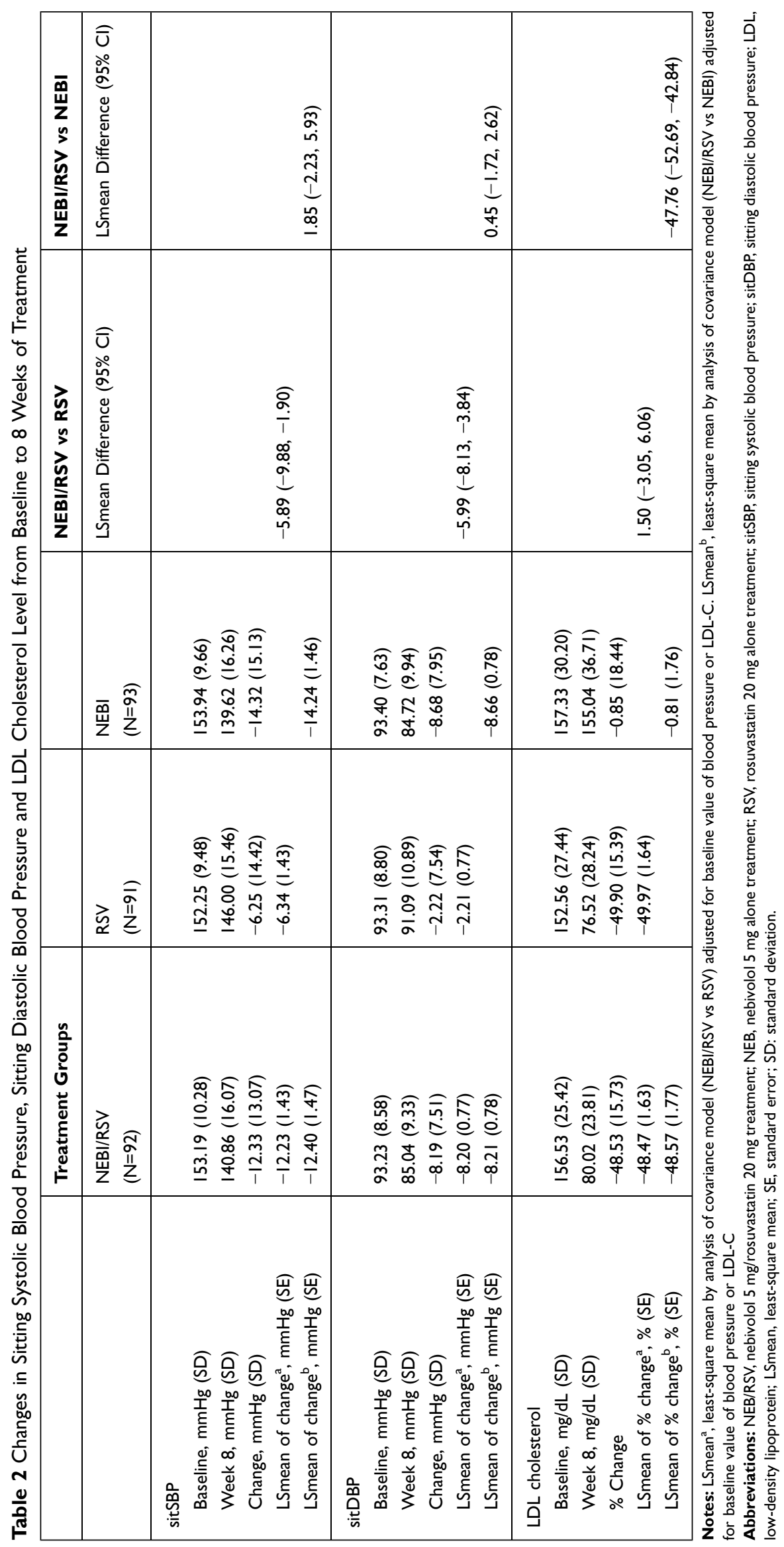



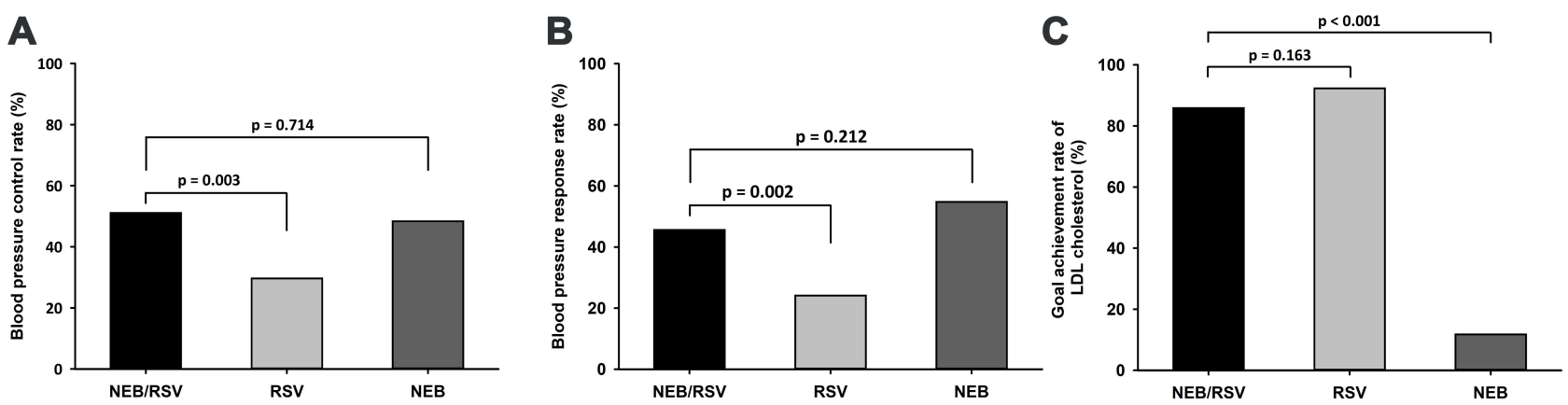

Figure 2 (A) Control rate and (B) response rate of blood pressure, and (C) goal achievement rate of low-density lipoprotein (LDL) cholesterol after 5 mg nebivolol plus $20 \mathrm{mg}$ rosuvastatin (NEBI/RSV), $20 \mathrm{mg}$ rosuvastatin (RSV), or $5 \mathrm{mg}$ nebivolol (NEBI) treatment for 8 weeks.

the NEBI/RSV group and four cases in the NEBI group. However, these patients recovered after discontinuing the study drugs. Heart rate was significantly decreased in the $\mathrm{NEBI} / \mathrm{RSV}$ (from $72.51 \pm 9.25 \mathrm{bpm}$ to $62.46 \pm 8.38 \mathrm{bpm}$, $p<0.001$ ) and NEBI (from $73.00 \pm 11.35 \mathrm{bpm}$ to $63.13 \pm$ $9.30 \mathrm{bpm}, p<0.001)$ groups, but was not changed in the RSV group (from $74.01 \pm 10.55 \mathrm{bpm}$ to $73.40 \pm 9.10 \mathrm{bpm}$, $p=0.639$ ). One patient had musculoskeletal pain (chest pain and tenderness, neck stiffness and shoulder pain). Chest pain and tenderness, and neck stiffness were improved with analgesics and were classified as unlikely when evaluating the relevance to the study drugs. Shoulder pain that occurred at a later time could not be classified owing to low adherence to study drugs (7.7\%). Two patients had dizziness that was resolved by discontinuing the study drugs.

There were no significant changes in fasting blood glucose levels from baseline after 8 weeks of treatment in the $\mathrm{NEBI} / \mathrm{RSV}$ (mean change $\pm \mathrm{SD}=0.68 \pm 17.99, p=0.763$ ), RSV (mean change $\pm \mathrm{SD}=-0.34 \pm 12.34, p=0.792$ ), and NEBI (mean change $\pm \mathrm{SD}=1.70 \pm 30.09, p=0.263$ ) groups. HbA1C levels were not significantly changed from baseline after 8 weeks of treatment in the NEBI/ $\mathrm{RSV}$ (mean change $\pm \mathrm{SD}=0.02 \pm 0.35, p=0.912$ ), RSV (mean change $\pm \mathrm{SD}=-0.03 \pm 0.48, p=0.679$ ), and NEBI (mean change $\pm \mathrm{SD}=-0.05 \pm 0.48, p=0.135$ ) groups.

\section{Discussion}

This is the first controlled, prospective study showing that nebivolol plus rosuvastatin treatment is effective and safe in patients with hypertension and hypercholesterolemia, without the loss of BP- or the LDL cholesterol-lowering effect of each drug. BP reduction by Nebivolol plus rosuvastatin treatment was not different from that by nebivolol alone treatment, and LDL cholesterol reduction by nebivolol plus rosuvastatin treatment was not different from that by rosuvastatin alone treatment. Nebivolol treatment either with rosuvastatin or alone significantly decreased the heart rate; the treatments also caused symptomatic bradycardia in few participants. However, the overall incidence of study drug-related AEs did not differ among the three groups.

Hypercholesterolemia and hypertension commonly coexist and synergistically contribute to cardiovascular disease. ${ }^{6,15}$ Therefore, both diseases should be controlled simultaneously. However, in many patients, high BP and LDL cholesterol levels are not controlled simultaneously, partly by inadequate doses of statins used. ${ }^{16}$ In our study, the percent change and goal achievement rate for LDL cholesterol levels were $48.5 \%$ and $85.9 \%$, respectively, in the NEBI/RSV group. Recent guidelines recommend more intense LDL cholesterol reduction for high and very highrisk patients (eg, LDL cholesterol $<70 \mathrm{mg} / \mathrm{dl}$ for high risk and $<55 \mathrm{mg} / \mathrm{dl}$ for very high risk), ${ }^{4}$ which, if applied, would decrease the goal achievement rate in our study. However, the percent reduction and goal achievement rate of our study indicate that high-intensity statin treatment may further improve the LDL cholesterol control rate in the real world, thus helping to prevent cardiovascular diseases.

A recent meta-analysis corroborated the inferiority of beta-blockers in reducing cardiovascular disease and mortality compared with other antihypertensive drugs, such as calcium channel blockers, renin-angiotensin-aldosterone inhibitors, and diuretics. ${ }^{17}$ However, most studies included in the meta-analysis used atenolol, a non-vasodilating beta1 selective blocker, which was inferior to angiotensin receptor blockers ${ }^{18}$ and calcium channel blockers. ${ }^{19-21}$ Nebivolol is a third-generation beta-blocker with unique properties, ${ }^{9}$ and such third-generation vasodilating beta-blockers (eg, 


\begin{tabular}{|c|c|c|c|c|c|c|}
\hline 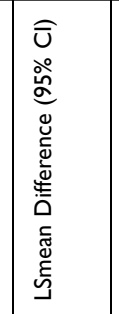 & 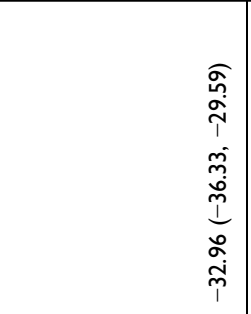 & 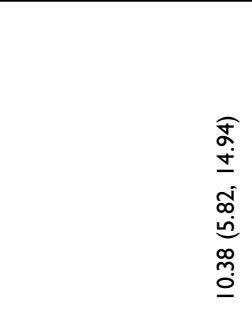 & 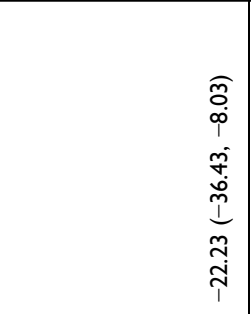 & 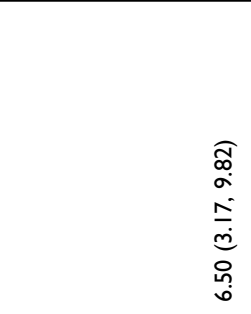 & 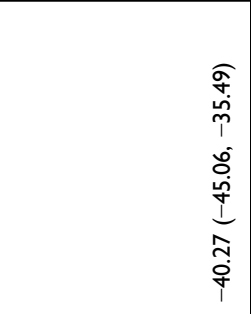 & \\
\hline 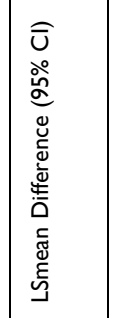 & 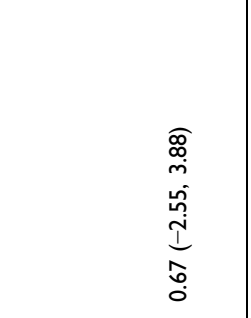 & 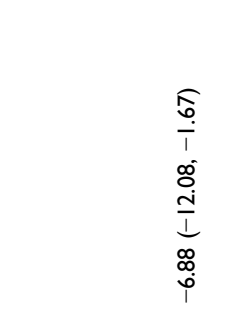 & 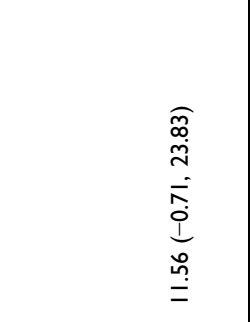 & 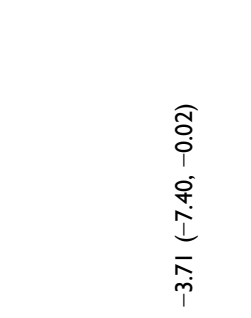 & 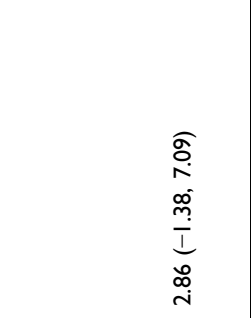 & \\
\hline 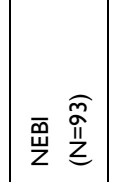 & 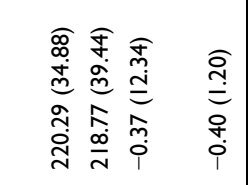 & 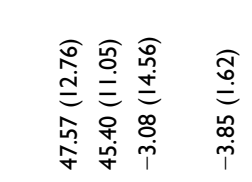 & 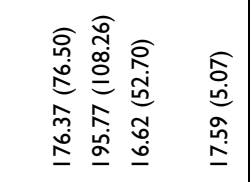 & 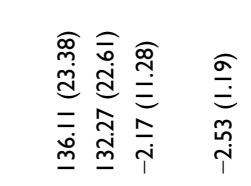 & 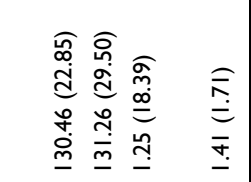 & 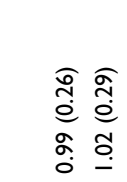 \\
\hline 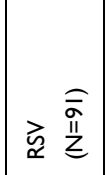 & 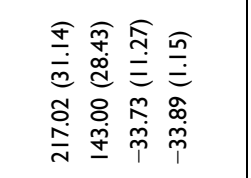 & 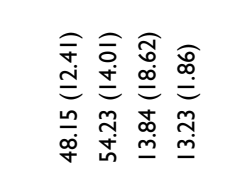 & 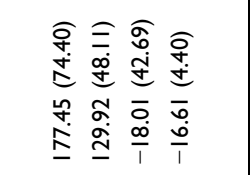 & 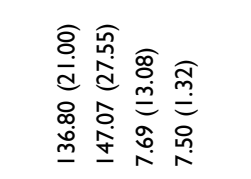 & 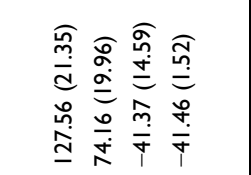 & 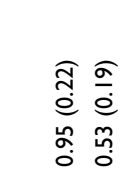 \\
\hline 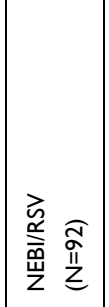 & 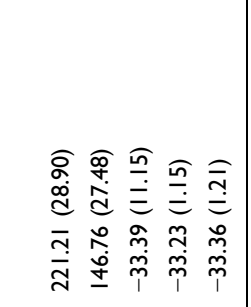 & 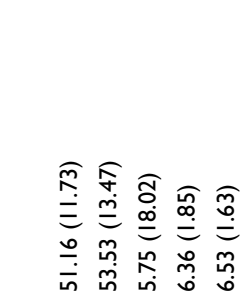 & 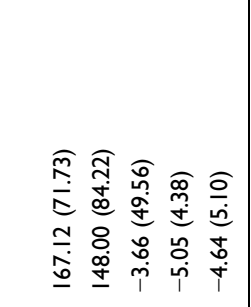 & 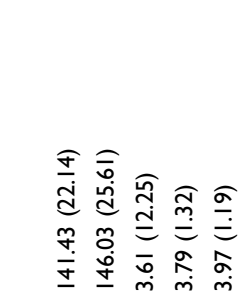 & 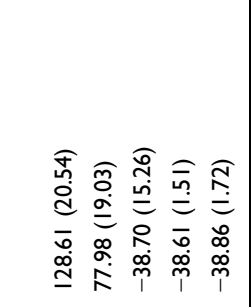 & 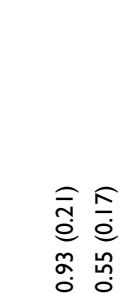 \\
\hline & 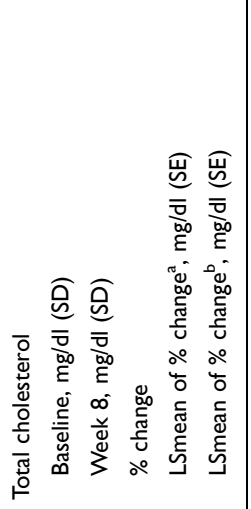 & 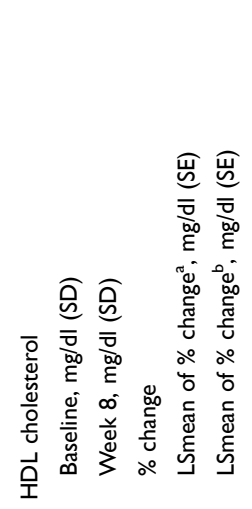 & 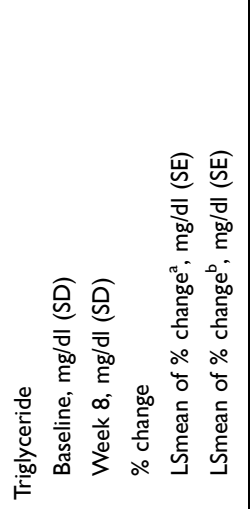 & 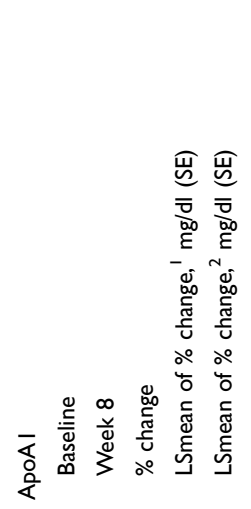 & 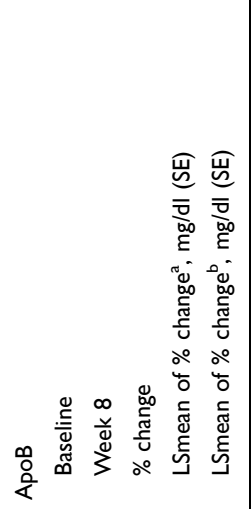 & 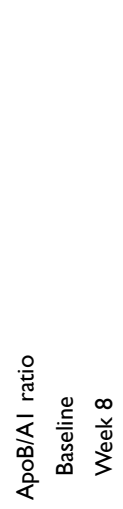 \\
\hline
\end{tabular}




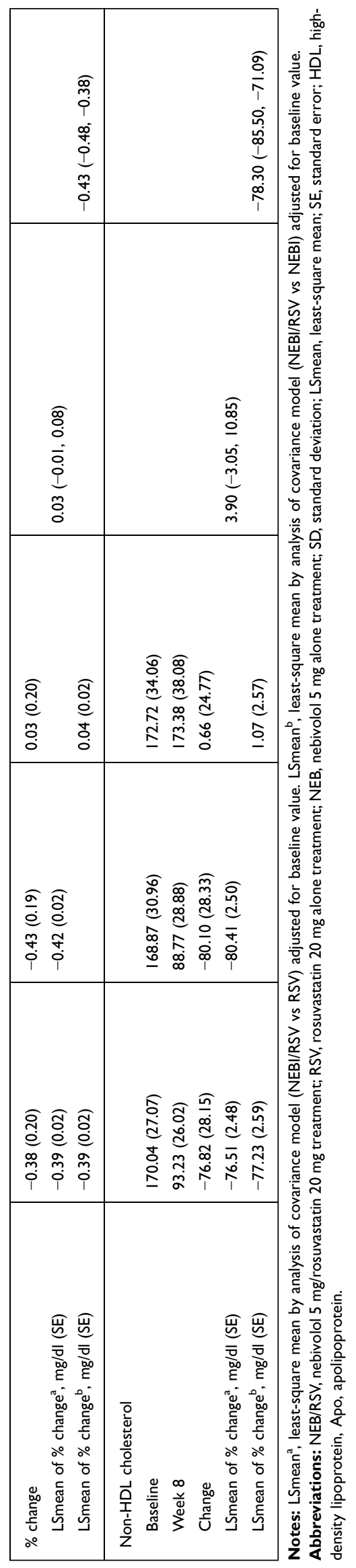

carvedilol and nebivolol) were not included in the above meta-analysis because outcome trials on hypertension were lacking. ${ }^{17}$ Although there are no clinical outcome trials, nebivolol is expected to perform better than atenolol in cardiovascular protection because of its vasodilatory property ${ }^{9}$ and long action period, aspect that differs from those of atenolol. ${ }^{22}$

Beta-blockers can dysregulate lipid metabolism and increase insulin resistance and susceptibility to diabetes. ${ }^{23,24}$ Moreover, ASCOT (Anglo-Scandinavian Outcomes Trial) results suggested that beta-blockers (atenolol) might attenuate the benefits of statin treatment and increase the rate of ischemic events compared to a combined calcium channel blocker and statin treatment. ${ }^{25}$ In contrast to traditional beta-blockers, nebivolol has a neutral or favorable effect on the metabolic profile. ${ }^{7,9}$ Before our study, randomized controlled studies that examined the effect of nebivolol treatment on lipid metabolism were sparse and with a small sample size. Moreover, no study had evaluated whether nebivolol impairs the beneficial effects of rosuvastatin on lipid metabolism.

In the present study, nebivolol treatment did not offset the effects of rosuvastatin in lowering LDL cholesterol level, ApoB, and the ApoB/A1 ratio. In contrast, nebivolol treatment reduced HDL cholesterol levels and increased triglyceride levels. Three previous studies have also reported a neutral effect of nebivolol on HDL cholesterol levels, ${ }^{26-28}$ and two studies even reported a reduction in HDL cholesterol levels. ${ }^{29,30}$ In the present study, nebivolol treatment lowered HDL cholesterol levels and seemed to attenuate the effect of rosuvastatin on increasing HDL cholesterol levels. Reasons for the different results among studies could not be clarified as the treatment schedule and inclusion criteria were quite different.

Reportedly, the level of HDL cholesterol has been inversely correlated with the risk of atherosclerotic cardiovascular disease (ASCVD). ${ }^{31}$ However, further studies are required to assess whether the undesirable lowering of HDL cholesterol levels by nebivolol observed in our study may attenuate the beneficial effect of rosuvastatin on ASCVD risks. Among the previous studies, ${ }^{26-30}$ none reported a significant elevation in triglyceride levels via nebivolol treatment. There was no difference in the reduction in triglycerides between NEBI/RSV and RSV groups. In contrast, those in the NEBI group had significantly elevated triglyceride levels. Elevated triglycerides are a risk factor for cardiovascular morbidity and all-cause 
Table 4 Adverse Drug Reaction in the Safety Analysis Set $(n=28 I)$

\begin{tabular}{|c|c|c|c|}
\hline & $\begin{array}{l}\text { NEB/RSV } \\
(n=94)\end{array}$ & $\begin{array}{l}\text { RSV } \\
(n=94)\end{array}$ & $\begin{array}{l}\text { NEB } \\
(n=93)\end{array}$ \\
\hline Number of subjects (\%) & $8(8.51 \%)$ & $7(7.45 \%)$ & $8(8.60 \%)$ \\
\hline Adverse drug reactions, total number of events & 8 & 11 & II \\
\hline Bradycardia & $2(2.13 \%)$ & & $4(4.30 \%)$ \\
\hline Atrial fibrillation & & I (I.06\%) & \\
\hline Ventricular premature beats & & $\mathrm{I}(\mathrm{I} .06 \%)$ & \\
\hline Increase in creatine phosphokinase & $2(2.13 \%)$ & $4(4.26 \%)$ & I (I.08\%) \\
\hline Increase in body weight & & I (I.06\%) & \\
\hline Headache & & $3(3.19 \%)$ & \\
\hline Dizziness & I (I.06\%) & & $\mathrm{I}(\mathrm{I} .08 \%)$ \\
\hline Diarrhea & & & $\mathrm{I}(\mathrm{I} .08 \%)$ \\
\hline Epigastric discomfort & I (I.06\%) & & \\
\hline Gastrointestinal disorder & & & $\mathrm{I}(\mathrm{I} .08 \%)$ \\
\hline Hyperglycemia & & & $\mathrm{I}(\mathrm{I} .08 \%)$ \\
\hline Hyperkalemia & I (I.06\%) & & \\
\hline Blurred vision & & I (I.06\%) & \\
\hline Thirst & & & $\mathrm{I}(\mathrm{I} .08 \%)$ \\
\hline Musculoskeletal pain & I (I.06\%) & & \\
\hline Cough & & & $\mathrm{I}(\mathrm{I} .08 \%)$ \\
\hline
\end{tabular}

Note: Adverse drug reactions are expressed as number of events and percentages based on the subjects within each treatment group.

Abbreviations: NEB/RSV, nebivolol $5 \mathrm{mg} /$ rosuvastatin $20 \mathrm{mg}$ treatment; NEB, nebivolol $5 \mathrm{mg}$ alone treatment; RSV, rosuvastatin $20 \mathrm{mg}$ alone treatment.

mortality. $^{32}$ Retaining ApoB-containing lipoproteins within the arterial intima is a major factor leading to the onset and development of atherosclerosis. ${ }^{33}$ Moreover, the risks of ASCVD mediated via triglycerides appear to be determined from the level of circulating ApoB-containing particles rather than triglyceride levels. ${ }^{34}$ In this study, as elevated triglycerides were not accompanied by changes in LDL cholesterol and ApoB levels and ApoB/A1 ratio, our findings suggest that elevating triglycerides via nebivolol treatment may not be associated with increased risks for ASCVD and does not offset the beneficial effect of rosuvastatin on the reduction of risks for ASCVD.

Long-term statin treatment increases the risk of newonset diabetes. ${ }^{35}$ Conversely, nebivolol has favorable effects on glucose and insulin resistance. ${ }^{27,29}$ Neither the RSV nor the NEBI/TSV groups displayed an increase in fasting blood glucose and $\mathrm{HbA1C}$ levels in this study. However, a long-term, large cohort study on the effects of combining nebivolol and rosuvastatin treatment on blood glucose and HbA1C levels is required.

For patients with hypertension, poor adherence to treatment is associated with increased rates of hospitalization and mortality. ${ }^{36}$ Among the methods to improve treatment adherence, using single-pill combination drugs has been shown to improve patients adherence to treatment by simplifying regimens and reducing pill burden. ${ }^{6,37}$ Therefore, single-pill combination drugs are now recommended in the guidelines. ${ }^{5,8}$ This study showed that combining nebivolol and rosuvastatin did not alter the beneficial BP- or the LDL cholesterol-lowering effects of each drug; therefore, this combination treatment could be used as a single-pill combination drug.

\section{Strength and Limitations}

Our study is the first to compare the effects of nebivolol plus rosuvastatin treatment on lipid parameters to those of nebivolol and rosuvastatin alone treatment. Previously, it remained unclear whether combining a vasodilatory betablocker with a statin would attenuate the beneficial effect of a statin on lipid parameters. Further, our study cohort was the largest among those in prospective and controlled studies evaluating the metabolic effects of nebivolol reported to date.

However, there are a few limitations to this study. First, age differences in the BP-lowering effects among different classes of antihypertensive drugs were not considered in the study design. Beta-blockers are considered to be more effective in lowering the BP in young hypertensive patients. $^{38}$ However, BP-lowering effects observed in the NEBI/RSV group did not differ between older ( $\geq 65$ years) 
and younger ( $<65$ years) patients. Although there is no previous study to compare these findings with, these results may be explained by the difference in the vasodilatory actions of nebivolol and bisoprolol. ${ }^{39}$ Second, this was a short-term study; the efficacy in terms of cardiovascular outcome was not evaluated. Whether favorable effects of nebivolol on glucose metabolism and insulin resistance offset the risk of new-onset diabetes via statin treatment could not be determined. Large-scale, long-term outcome studies for the efficacy of nebivolol plus statin combination treatment comparing with that of other antihypertensive drugs plus statin for treatment and prevention of cardiovascular disease are required.

\section{Conclusion}

This study demonstrated that the BP- and LDL cholesterol-lowering efficacy and safety of combining nebivolol plus rosuvastatin treatment are comparable with those of nebivolol and rosuvastatin alone treatment in patients with hypertension and hypercholesterolemia. The results of this study indicate that nebivolol plus rosuvastatin could be used without the loss of BP or the LDL cholesterollowering effect of each drug and an acceptable combination in treating and preventing cardiovascular diseases.

\section{Abbreviations}

CI, confidence interval; AEs, adverse events; ANCOVA, analysis of covariance; ASCVD, atherosclerotic cardiovascular disease; BP, blood pressure; bpm, beats per minute; FAS, full analysis set; LDL, low-density lipoprotein; LSmean, least-square mean; NCEP-ATP III, National Cholesterol Education Program Adult Panel III; NEBI/ $\mathrm{RSV}$, co-administration of nebivolol $5 \mathrm{mg}$ and rosuvastatin $20 \mathrm{mg}$; NEBI, nebivolol $5 \mathrm{mg}$ alone treatment; PPS, perprotocol set; RSV, rosuvastatin $20 \mathrm{mg}$ alone treatment; SAF, safety analysis set; SD, standard deviation; sitDBP, sitting diastolic blood pressure; sitSBP, sitting systolic blood pressure; TLC, therapeutic lifestyle change.

\section{Data Sharing Statement}

We are not planning to share the data besides what is included in the manuscript.

\section{Acknowledgments}

Investigators: Kyung Heon Won (Seoul Medical Center), Kiyuk Chang (The Catholic University of Korea Seoul St. Mary's Hospital), Bong-Ki Lee (Kangwon National University Hospital), Sang Hoon Kim (CHA Bundang
Medical Center), Dong-Bin Kim (The Catholic University of Korea St. Paul's Hospital), Jinho Shin (Hanyang University Hospital), Kyoo-Rok Han (Kangdong Sacred Heart Hospital), Cheol Whan Lee (Asan Medical Center), Jung Hyun Choi (Pusan National University Hospital), GeeHee Kim (The Catholic University of Korea St. Vincent's Hospital), Hyungseop Kim (Keimyung University Dongsan Medical Center), Tae-Ho Park (Dong-A University Hospital), Jin-Ok Jeong (Chungnam National University Hospital), Jin Bae Lee (Daegu Catholic University Medical Center), Sung-Ho Her (The Catholic University of Korea Daejeon St. Mary's Hospital), Jeong Cheon Ahn (Korea University Ansan Hospital), Soo-Joong Kim (Kyung Hee University Hospital), Sang Kyoon Cho (Bundang Jesaeng Hospital), Dong Woon Jeon (National Health Insurance Service Ilsan Hospital), and Bum-Kee Hong (Gangnam Severance Hospital).

\section{Author Contributions}

All authors made a significant contribution to the work reported, whether that is in the conception, study design, execution, acquisition of data, analysis and interpretation, or in all these areas; took part in drafting, revising or critically reviewing the article; gave final approval of the version to be published; have agreed on the journal to which the article has been submitted; and agree to be accountable for all aspects of the work.

\section{Funding}

The primary study was initiated and supported financially by Elyson Pharmaceutical Co., LTD. The company was involved in all stages of the study conduct and design. Elyson Pharmaceutical Co., LTD. also took responsibility for all costs associated with the development and publishing of the manuscript. This research was supported by the Ministry of Trade, Industry and Energy (Korea), the Korea Evaluation Institute of Industrial Technology (Korea) grant (No.10052298).

\section{Disclosure}

M.Y. Rhee has received lecture honoraria from Pfizer Inc., LG Life Sciences Ltd., Boehringer Ingelheim Pharma GmbH \& Co. KG., Hanmi Pharm. Co. Ltd., Yuhan Co. Ltd., and Boryung Pharmaceutical Co. Ltd.; consulting fees from Hanmi Pharm. Co. Ltd. and Shin Poong Pharma. Co. Ltd.; research grants from Boryung Pharmaceutical Co. Ltd. and Dong-A Pharmaceutical Co. Ltd.; and reports personals fees from Pfizer Inc. and 
consulting fees from Hanmi Pharm. Co. Ltd and Shin Poong Pharma. Co. Ltd, during the conduct of the study.

Y. Ahn has received research grants from Medtronic Korea, Boston Scientific corporation, Abbott Corporation, and Qualitech Korea.

C.H. Kim has received lecture honoraria from GlaxoSmithKline and Hanmi Pharmaceutical Co. Ltd and research grant from Merck Sharp \& Dohme, LG Life Sciences Ltd., and Boryung Pharmaceutical Co. Ltd.

The aforementioned authors report no other conflicts of interest and the remaining authors have indicated that they have no conflicts of interest with regard to this work.

\section{References}

1. Johnson ML, Pietz K, Battleman DS, Beyth RJ. Prevalence of comorbid hypertension and dyslipidemia and associated cardiovascular disease. Am J Manag Care. 2004;10:926-932.

2. Jackson R, Lawes CM, Bennett DA, Milne RJ, Rodgers A. Treatment with drugs to lower blood pressure and blood cholesterol based on an individual's absolute cardiovascular risk. Lancet. 2005;365:434-441. doi:10.1016/S0140-6736(05)70240-3

3. Emberson J. Evaluating the impact of population and high-risk strategies for the primary prevention of cardiovascular disease. Eur Heart J. 2004;25(6):484-491. doi:10.1016/j.ehj.2003.11.012

4. Mach F, Baigent C, Catapano AL, et al. 2019 ESC/EAS Guidelines for the management of dyslipidaemias: lipid modification to reduce cardiovascular risk. Eur Heart J. 2019.

5. Whelton PK, Carey RM, Aronow WS, et al. 2017 ACC/AHA/AAPA/ ABC/ACPM/AGS/APhA/ASH/ASPC/NMA/PCNA guideline for the prevention, detection, evaluation, and management of high blood pressure in adults: a report of the american college of cardiology/ american heart association task force on clinical practice guidelines. Hypertension. 2018;71:e13-e115.

6. Hypertension in adults: diagnosis and management. NICE guideline [NG136]. National institute for health and care excellence; Published 2019. Available from: https://www.nice.org.uk/guidance/ng136. Accessed March 13, 2020.

7. Marketou M, Gupta Y, Jain S, Vardas P. Differential metabolic effects of beta-blockers: an updated systematic review of nebivolol. Curr Hypertens Rep. 2017;19:22. doi:10.1007/s11906-017-0716-3

8. Williams B, Mancia G, Spiering W, et al. ESC/ESH Guidelines for the management of arterial hypertension. Eur Heart J. 2018;39:3021-3104.

9. Munzel T, Gori T. Nebivolol: the somewhat-different beta-adrenergic receptor blocker. J Am Coll Cardiol. 2009;54:1491-1499. doi:10.1016/j.jacc.2009.05.066

10. National Cholesterol Education Program (NCEP). Expert Panel on Detection, Evaluation, and Treatment of High Blood Cholesterol in Adults (Adult Treatment Panel III). Third Report of the National Cholesterol Education Program (NCEP) Expert Panel on Detection, Evaluation, and Treatment of High Blood Cholesterol in Adults (Adult Treatment Panel III) final report. Circulation. 2002;106(25):3143-3421.

11. Weiss RJ, Weber MA, Carr AA, Sullivan WA. A randomized, double-blind, placebo-controlled parallel-group study to assess the efficacy and safety of nebivolol, a novel beta-blocker, in patients with mild to moderate hypertension. J Clin Hypertens. 2007;9:667-676. doi:10.1111/j.1524-6175.2007.06679.x

12. Giles TD, Khan BV, Lato J, Brener L, Ma Y, Lukic T. Nebivolol monotherapy in younger adults (younger than 55 years) with hypertension: a randomized, placebo-controlled trial. J Clin Hypertens. 2013;15:687-693. doi:10.1111/jch.12169
13. McKenney JM. Efficacy and safety of rosuvastatin in treatment of dyslipidemia. Am J Health Syst Pharm. 2005;62:1033-1047. doi:10.1093/ajhp/62.10.1033

14. Saito Y, Goto Y, Dane A, Strutt K, Raza A. Randomized dose-response study of rosuvastatin in Japanese patients with hypercholesterolemia. $J$ Atheroscler Thromb. 2003;10:329-336. doi: $10.5551 /$ jat. 10.329

15. Neaton JD, Wentworth D. Serum cholesterol, blood pressure, cigarette smoking, and death from coronary heart disease. Overall findings and differences by age for 316,099 white men. Multiple risk factor intervention trial research group. Arch Intern Med. 1992;152:56-64. doi:10.1001/archinte.1992.00400130082009

16. Spannella F, Giulietti F, Di Pentima C, Sarzani R. Prevalence and control of dyslipidemia in patients referred for high blood pressure: the disregarded "double-trouble" lipid profile in overweight/obese. Adv Ther. 2019;36:1426-1437. doi:10.1007/s12325-019-00941-6

17. Szyndler A, Stolarz-Skrzypek K, Kuznetsova T, et al. Fatal and nonfatal outcomes, incidence of hypertension, and blood pressure changes in relation to urinary sodium excretion. JAMA, 2011; 305: 1777-1785]. Kardiol Pol. 2011;69:1314-1315.

18. Dahlof B, Devereux RB, Kjeldsen SE, et al. Cardiovascular morbidity and mortality in the losartan intervention for endpoint reduction in hypertension study (LIFE): a randomised trial against atenolol. Lancet. 2002;359:995-1003. doi:10.1016/S0140-6736(02)08089-3

19. Zanchetti A, Bond MG, Hennig M, et al. Calcium antagonist lacidipine slows down progression of asymptomatic carotid atherosclerosis: principal results of the European lacidipine study on atherosclerosis (ELSA), a randomized, double-blind, long-term trial. Circulation. 2002;106:2422-2427. doi:10.1161/01. CIR.0000039288.86470.DD

20. Pepine CJ, Handberg EM, Cooper-DeHoff RM, et al. A calcium antagonist vs a non-calcium antagonist hypertension treatment strategy for patients with coronary artery disease. The International Verapamil-Trandolapril Study (INVEST): a randomized controlled trial. JAMA. 2003;290:2805-2816. doi:10.1001/jama.290.21.2805

21. Dahlof B, Sever PS, Poulter NR, et al. Prevention of cardiovascular events with an antihypertensive regimen of amlodipine adding perindopril as required versus atenolol adding bendroflumethiazide as required, in the Anglo-Scandinavian Cardiac Outcomes Trial-Blood Pressure Lowering Arm (ASCOT-BPLA): a multicentre randomised controlled trial. Lancet. 2005;366:895-906.

22. Van Nueten L, Dupont AG, Vertommen C, Goyvaerts H, Robertson JI. A dose-response trial of nebivolol in essential hypertension. J Hum Hypertens. 1997;11:139-144. doi:10.1038/sj. jhh. 1000392

23. Cooper-Dehoff R, Cohen JD, Bakris GL, et al. Predictors of development of diabetes mellitus in patients with coronary artery disease taking antihypertensive medications (findings from the INternational VErapamil SR-Trandolapril STudy [INVEST]). Am J Cardiol. 2006;98:890-894. doi:10.1016/j.amjcard.2006.04.030

24. Elliott WJ, Meyer PM. Incident diabetes in clinical trials of antihypertensive drugs: a network meta-analysis. Lancet. 2007;369:201-207. doi:10.1016/S0140-6736(07)60108-1

25. Sever P, Dahlof B, Poulter N, et al. Potential synergy between lipid-lowering and blood-pressure-lowering in the anglo-scandinavian cardiac outcomes trial. Eur Heart J. 2006;27:2982-2988. doi:10.1093/eurheartj/ehl403

26. Fogari R, Zoppi A, Lazzari P, et al. Comparative effects of nebivolol and atenolol on blood pressure and insulin sensitivity in hypertensive subjects with type II diabetes. J Hum Hypertens. 1997;11:753-757. doi:10.1038/sj.jhh.1000533

27. Rizos E, Bairaktari E, Kostoula A, et al. The combination of nebivolol plus pravastatin is associated with a more beneficial metabolic profile compared to that of atenolol plus pravastatin in hypertensive patients with dyslipidemia: a pilot study. J Cardiovasc Pharmacol Ther. 2003;8:127-134. doi:10.1177/107424840300800206 
28. Lacourciere Y, Poirier L, Lefebvre J, Provencher P, Arnott W. Comparative effects of a new cardioselective beta-blocker nebivolol and nifedipine sustained-release on 24-hour ambulatory blood pressure and plasma lipoproteins. J Clin Pharmacol. 1992;32:660-666. doi:10.1002/j.1552-4604.1992.tb05778.x

29. Ozyildiz AG, Eroglu S, Bal U, Atar I, Okyay K, Muderrisoglu H. Effects of carvedilol compared to nebivolol on insulin resistance and lipid profile in patients with essential hypertension. J Cardiovasc Pharmacol Ther. 2017;22:65-70. doi:10.1177/1074248416644987

30. Pesant Y, Marc-Aurele J, Bielmann P, et al. Metabolic and antihypertensive effects of nebivolol and atenolol in normometabolic patients with mild-to-moderate hypertension. Am J Ther. 1999;6:137-147. doi:10.1097/00045391-199905000-00004

31. Lewington S, Whitlock G, Clarke R, et al. Blood cholesterol and vascular mortality by age, sex, and blood pressure: a meta-analysis of individual data from 61 prospective studies with 55,000 vascular deaths. Lancet. 2007;370:1829-1839.

32. Nordestgaard BG, Varbo A. Triglycerides and cardiovascular disease. Lancet. 2014;384:626-635. doi:10.1016/S0140-6736(14)61177-6

33. Boren J, Williams KJ. The central role of arterial retention of cholesterol-rich apolipoprotein-B-containing lipoproteins in the pathogenesis of atherosclerosis: a triumph of simplicity. Curr Opin Lipidol. 2016;27:473-483. doi:10.1097/MOL.0000000000000330
34. Laufs U, Parhofer KG, Ginsberg HN, Hegele RA. Clinical review on triglycerides. Eur Heart J. 2020;41:99-109c. doi:10.1093/eurheartj/ ehz785

35. Sattar N, Preiss D, Murray HM, et al. Statins and risk of incident diabetes: a collaborative meta-analysis of randomised statin trials. Lancet. 2010;375:735-742. doi:10.1016/S0140-6736(09)61965-6

36. Kim S, Shin DW, Yun JM, et al. Medication adherence and the risk of cardiovascular mortality and hospitalization among patients with newly prescribed antihypertensive medications. Hypertension. 2016;67:506-512. doi:10.1161/HYPERTENSIONAHA.115.06731

37. Bangalore S, Kamalakkannan G, Parkar S, Messerli FH. Fixed-dose combinations improve medication compliance: a meta-analysis. $\mathrm{Am}$ J Med. 2007;120:713-719. doi:10.1016/j.amjmed.2006.08.033

38. Williams B, Poulter NR, Brown MJ, et al. Guidelines for management of hypertension: report of the fourth working party of the british hypertension society, 2004-BHS IV. J Hum Hypertens. 2004;18:139-185.

39. Simova II, Todorova-Konstantinova RR, Denchev SV. Effects of nebivolol versus bisoprolol on endothelial function in hypertensive patients. Exp Clin Cardiol. 2009;14:45-49.
Drug Design, Development and Therapy

\section{Publish your work in this journal}

Drug Design, Development and Therapy is an international, peerreviewed open-access journal that spans the spectrum of drug design and development through to clinical applications. Clinical outcomes, patient safety, and programs for the development and effective, safe, and sustained use of medicines are a feature of the journal, which has also

\section{Dovepress}

been accepted for indexing on PubMed Central. The manuscript management system is completely online and includes a very quick and fair peer-review system, which is all easy to use. Visit http://www. dovepress.com/testimonials.php to read real quotes from published authors. 